\title{
SISTEMA ABO E FORMAS ANATOMOCLÍNICAS DA DOENÇA DE CHAGAS CRÔNICA
}

\section{Vicente de Paula Antunes Teixeira, Elizabeth Martins, Hipolito de Oliveira Almeida, Sheila Soares, Hélio Moraes de Souza e César Augusto de Morais}

\begin{abstract}
Estudou-se a distribuição do sistema ABO em 222 controles e em 148 chagásicos crônicos, assintomáticos e sintomáticos, com diferentes formas anatomoclínicas (insuficiência cardiaca congestiva, visceromegalias e morte súbita). $O X^{2}$ foi calculado a partir de tabelas de contingência e pelo método de Woolf. $O X^{2}$, obtido por estes dois métodos, demonstra que a freqüência do grupo O foi significativamente menor nos chagásicos sintomáticos considerados como um todo e naqueles com "megas", em relação aos controles. Nos chagásicos falecidos subitamente observou-se maior freqüência de grupos $B$ que nos controles, sendo o $X^{2}$ significativo apenas quando calculado por tabelas de contingência. A relativa proteção aparentemente conferida aos chagásicos do grupo $O$, no que se refere à evolução para formas sintomáticas da doença, se explicaria por antigenicidade cruzada entre populaçôes do Trypanosoma cruzi $e$ o sistema $A B O$ ou por outros fatores que se deixam influenciar ou influenciam a distribuição dos grupos sangüineos.
\end{abstract}

Palavras chaves: Doença de Chagas. Grupos sangüineos. Sistema ABO.

As doenças humanas produzidas por agentes biológicos (virus, bactérias, fungos, protozoários) têm suas flutuações e seus polimorfismos anatomoclínicos com diferentes respostas terapêuticas, determinadas por uma multiplicidade de fatores do meio ambiente (umidade, temperatura, alimentação, poluentes, luz, etc.) e por variações do agente biológico agressor que podem modificar-lhe tropismos e ação patogênica. Entretanto, as caracteristicas do organismo humano agredido são importantes para bloquear, permitir, reduzir, exacerbar ou modificar qualitativamente as ações e tropismos patogênicos de um agente biológico que o invade. Muitas destas caracteristicas são geneticamente determinadas, sendo responsáveis por variações nos mecanismos de defesa. A identificação de pessoas ou populações mais resistentes ou mais sensiveis a agressão por certos agentes tem importância em medicina preventiva e auxilia no raciocínio clínico como dado estatístico para orientar a formulação do diagnóstico e avaliação prognóstica. Dentre os marcadores genéticos que podem fornecer elementos para o raciocínio clínico, estão os sistemas antigênicos HLA e ABO. A identificação de antígenos do sistema $A B O$, pela sua simplicidade e por ser de baixo custo, vem sendo empregada há algumas décadas permitindo analisar a relação dos diferentes grupos sanguíneos com infecções bacterianas ${ }^{11} 12$, viróticas 6 , por alguns protozoários ${ }^{4}$, esquistossomose ${ }^{2} 37$, além de outras doenças não infecciosas como mal-formações $^{11}$, tumores ${ }^{113}$ e úlcera péptica ${ }^{1}$. Apesar de

Disciplina de Patologia Geral e Serviço de Hematologia do Hospital-Escola da Faculdade de Medicina do Triângulo Mineiro, Uberaba-MG.

Recebido para publicação em 10/3/86. altamente endêmica em nosso meio, a doença de Chagas foi muito pouco explorada nesse sentido. Há alguns anos, as disciplinas de Patologia Geral e de Hematologia Clínica da Faculdade de Medicina do Triângulo Mineiro vêm, sistematicamente, fazendo tipagem do sistema $A B O$, pós-morte, no sangue e no liquido pericárdico, colhidos em necrópsias, além de realização das reações imunológicas para $T$. cruzi, nos mesmos materiais. No presente trabalho relatamos os resultados deste estudo, comparando a distribuição dos grupos sanguíneos nas populações de chagásicos e não chagásicos. Tal distribuição do sistema $\mathrm{ABO}$ é analisada na população de chagásicos como um todo $e$ subdividida em assintomáticos, vítimas de morte súbita, portadores de megas e portadores de ICC.

\section{MATERIAL E MÉTODOS}

A determinação do grupo sanguíneo foi realizada simultaneamente no liquido pericárdico (classificação reversa) e no sangue das cavidades cardiacas, ambos colhidos à necrópsia após abertura do saco pericárdico, utilizando-se seringas de vidro. $\mathrm{Na}$ classificação direta do sangue (feita em tubo) utilizou-se solução a 5\% das hemácias-problema e soros anti-A e anti-B da marca Biotest ${ }^{R}$. A classificação reversa do líquido pericárdico foi feita com o emprego de hemácias, (Revercel Biotest ${ }^{R}$ ) utilizando-se a técnica recomendada pelo fabricante para classificação reversa do soro. Foram desprezados todos os casos em que a tipagem do líquido pericárdico e do sangue deram resultados discordantes. Dos 370 casos estudados, 148 apresentaram reações imunológicas positivas para $T$. cruzi e 222 apresentaram reações negativas para doença de Chagas (controle). Os chagásicos 
Teixeira VPA, Martins E, Almeida HO, Soares S, Souza HM, Morais CA. Sistema ABO e formas anatomoclínicas da doença de Chagas crônica. Revista da Sociedade Brasileira de Medicina Tropical 20: 163-168, Jul-Set, 1987.

foram subdivididos em grupos, segundo os achados anatomoclínicos: a) pacientes, que apesar de ter as reações imunológicas positivas para $T$. cruzi, faleceram de causas não relacionadas com tripanosomose e não apresentavam manifestações clínicas, nem lesões compatíveis com o diagnóstico de ICC. Este grupo de chagásicos foi chamado assintomático; b) pacientes falecidos em decorrência de insuficiência cardíaca congestiva (ICC); c) portadores de megaesôfago e/ou megacólon; d) pacientes falecidos subitamente sem sinais de ICC ou de visceromegalias. A população de chagásicos como um todo é dividida em assintomáticos e sintomáticos, e estes, segundo as alterações anatomoclínicas, foram comparados com os controles, no que se refere às freqüências dos grupos sangǘneos $\mathrm{O}, \mathrm{A}, \mathrm{B}, \mathrm{AB}$. $\mathrm{A}$ análise estatística foi realizada através do $\mathrm{X}^{2}$ calculado através de tabelas de contingência $\left(X^{2}=\Sigma(0-e)^{2}\right)$ e segundo Woolf ${ }^{13}$. As diferenças observadas entre as populações analisadas foram consideradas significativas quando $\mathbf{P}<0,05$.

\section{RESULTADOS}

As Tabelas 1, 2, 3 e 4 mostram os resultados da análise estatística $\left(\mathrm{X}^{2}\right)$ pelo método de Woolf e por tabela de contingência, comparando os diferentes grupos de chagásicos com o grupo controle, no que se refere a grupos sanguineos $\mathrm{O}, \mathrm{A}, \mathrm{B}$ e $\mathrm{AB}$, respectivamente. A Tabela 5 mostra as freqüências dos diferentes grupos sangüíneos no grupo controle, nos chagásicos assintomáticos e sintomáticos.

Tabela 1 - Ocorrência de grupo $O$ em chagásicos e controles. As duas últimas colunas mostram qui-quadrado $\left(X^{2}\right)$ calculado segundo Woolf e por tabelas de contingência.

\begin{tabular}{|c|c|c|c|c|c|c|c|c|c|}
\hline \multirow[b]{3}{*}{ Chagásicos (total) } & \multicolumn{2}{|c|}{ Chagásicos } & \multicolumn{2}{|c|}{ Controle } & \multirow{2}{*}{$x=\frac{h K}{H k}$} & \multicolumn{2}{|c|}{ Cálculo de $x$ e $X^{2}$ segundo Woolf } & \multirow{2}{*}{$X^{2}=W y^{2}$} & \multirow{2}{*}{$\begin{array}{c}X^{2}= \\
\Sigma \frac{(o-e)^{2}}{e}\end{array}$} \\
\hline & $\begin{array}{r}\text { Grupo } \\
\text { (h) }\end{array}$ & $\begin{array}{r}\text { Não } \\
\text { (k) }\end{array}$ & $\begin{array}{r}\text { Grupo } \\
\text { (H) }\end{array}$ & $\begin{array}{c}\text { Não } O \\
(K)\end{array}$ & & $y=\log _{e} x$ & $W=\frac{1}{\frac{1}{h}+\frac{1}{k}+\frac{1}{H}+\frac{1}{K}}$ & & \\
\hline & 66 & 82 & 116 & 106 & 0,69 & $-0,31$ & 20,00 & 2,74 & 2,08 \\
\hline Assintomáticos & 40 & 35 & 116 & 106 & 1,04 & 0,04 & 12,50 & 0,02 & 0,02 \\
\hline Sintomáticos & 26 & 47 & 116 & 106 & 0,51 & $-0,67$ & 12,50 & $5,63\left({ }^{*}\right)$ & $6,09\left(^{*}\right)$ \\
\hline Mega & 10 & 21 & 116 & 106 & 0,44 & $-0,82$ & 5,88 & $3,94\left({ }^{*}\right)$ & $4,34\left({ }^{*}\right)$ \\
\hline$\overline{\mathrm{ICC}}$ & 11 & 19 & 116 & 106 & 0,53 & $-0,63$ & 6,25 & 2,50 & 2,26 \\
\hline Morte súbita & 5 & 7 & 116 & 106 & 0,66 & $-0,41$ & 2,8 & 0,47 & 0,52 \\
\hline
\end{tabular}

(*) Diferença significativa em relação ao controle $(P<0,05)$

Tabela 2 - Ocorrência de grupo A em chagásicos e controles. As duas últimas colunas mostram o qui-quadrado $\left(X^{2}\right)$ calculado segundo Woolf e por tabelas de contingência.

\begin{tabular}{|c|c|c|c|c|c|c|c|c|c|}
\hline \multirow[b]{3}{*}{ Chagásicos (total) } & \multicolumn{2}{|c|}{ Chagásicos } & \multicolumn{2}{|c|}{ Controle } & \multirow{2}{*}{$x=\frac{h K}{H k}$} & \multirow{2}{*}{$y=\log _{e} x$} & \multirow{2}{*}{$W=\frac{1}{\frac{1}{h}+\frac{1}{k}+\frac{1}{H}+\frac{1}{K}}$} & \multirow{2}{*}{$X^{2}=W y^{2}$} & \multirow{2}{*}{$\begin{array}{c}X^{2}= \\
\Sigma \frac{(o-e)^{2}}{e}\end{array}$} \\
\hline & $\begin{array}{c}\text { Grupo } A \\
\text { (h) }\end{array}$ & $\begin{array}{c}\text { Não } A \\
\text { (k) }\end{array}$ & $\begin{array}{c}\text { Grupo } A \\
(H)\end{array}$ & $\begin{array}{c}\text { Não A } \\
(K)\end{array}$ & & & & & \\
\hline & 54 & 94 & 78 & 144 & 1,06 & 0,06 & 20,00 & 0,07 & 0,07 \\
\hline Assintomáticos & 24 & 51 & 78 & 144 & 0,87 & $-0,14$ & 12,50 & 0,24 & 0,24 \\
\hline Sintomáticos & 30 & 43 & 78 & 144 & 1,29 & 0,25 & 14,29 & 0,89 & 0,84 \\
\hline Mega & 13 & 18 & 78 & 144 & 1,33 & 0,28 & 0,25 & 0,49 & 0,25 \\
\hline ICC & 14 & 16 & 78 & 144 & 1,62 & 0,55 & 6,67 & 2,02 & 1,51 \\
\hline Morte súbita & 3 & 9 & 78 & 144 & 0,41 & 0,89 & 2,19 & 1,73 & 0,52 \\
\hline
\end{tabular}


Teixeira VPA, Martins E, Almeida HO, Soares S, Souza HM, Morais CA. Sistema ABO e formas anatomoclínicas da doença de Chagas crônica. Revista da Sociedade Brasileira de Medicina Tropical 20: 163-168, Jul-Set, 1987.

Tabela 3 - Ocorrência de Grupo B em chagásicos e controles. As duas últimas colunas mostram o $X^{2}$ calculado segundo Woolf e por tabelas de contingência.

\begin{tabular}{|c|c|c|c|c|c|c|c|c|c|}
\hline \multirow[b]{3}{*}{ Chagásicos (total) } & \multicolumn{2}{|c|}{ Chagásicos } & \multicolumn{2}{|c|}{ Controle } & \multirow{2}{*}{$x=\frac{h K}{H k}$} & \multirow{2}{*}{$y=\log _{e} x$} & \multirow{2}{*}{$W=\frac{1}{\frac{l}{h}+\frac{l}{k}+\frac{1}{H}+\frac{l}{K}}$} & \multirow{2}{*}{$X^{2}=W y^{2}$} & \multirow{2}{*}{$\begin{array}{c}X^{2}= \\
\frac{\Sigma(o-e)^{2}}{e}\end{array}$} \\
\hline & $\begin{array}{c}\text { Grupo } \\
\text { (h) }\end{array}$ & $\begin{array}{c}N a \tilde{o} B \\
(k)\end{array}$ & $\begin{array}{r}\text { Grupo } \\
(H)\end{array}$ & $\begin{array}{c}\text { Nâo } B \\
(K)\end{array}$ & & & & & \\
\hline & 19 & 129 & 18 & 204 & 1,67 & 0,51 & 8,33 & 2,16 & 2,20 \\
\hline Assintomáticos & 8 & 67 & 18 & 204 & 1,35 & 0,30 & 5,00 & 0,45 & 0,46 \\
\hline Sintomáticos & 11 & 62 & 18 & 204 & 2,01 & 0,69 & 6,25 & 2,97 & 2,99 \\
\hline$\overline{\text { Mega }}$ & 4 & 27 & 18 & 204 & 1,68 & 0,52 & 2,86 & 0,77 & 0,79 \\
\hline$\overline{\mathrm{ICC}}$ & 4 & 26 & 18 & 204 & 1,74 & 0,55 & 2,86 & 0,86 & 0,901 \\
\hline Morte súbita & 3 & 9 & 18 & 204 & 3,77 & 1,32 & 2,00 & 3,49 & $4,01 *$ \\
\hline
\end{tabular}

* Diferença significativa em relaçāo aos controles $(P<0,05)$

Tabela 4 - Ocorrência de grupo $\mathrm{AB}$ em chagásicos e controles. As duas últimas colunas mostram qui-quadrado $\left(X^{2}\right)$ calculado segundo Woolf e por tabelas de contingência.

\begin{tabular}{|c|c|c|c|c|c|c|c|c|c|c|}
\hline & \multicolumn{2}{|c|}{ Chagásicos } & \multicolumn{2}{|c|}{ Controle } & \multirow{2}{*}{$x=\frac{h K}{H k}$} & \multirow{2}{*}{$y=\log _{e} x$} & \multirow{2}{*}{$\frac{W=}{h}+\frac{1}{k}$} & 1 & \multirow{2}{*}{$X^{2}=W y^{2}$} & \multirow{2}{*}{$\begin{array}{c}X^{2}= \\
\sum \frac{(0-e)^{2}}{e}\end{array}$} \\
\hline & $\begin{array}{l}\text { Grupo } A \\
\text { (h) }\end{array}$ & $\begin{array}{l}\operatorname{Vão} A b \\
(k)\end{array}$ & $\begin{array}{l}\text { Grupo } A \\
(H)\end{array}$ & $\begin{array}{c}\text { Não } A B \\
(K)\end{array}$ & & & & $\frac{+1}{H} \frac{+1}{K}$ & & \\
\hline Chagásicos (total) & 9 & 139 & 10 & 212 & 1,37 & 0,31 & & 4,55 & 0,44 & 0,45 \\
\hline Assintomáticos & 3 & 72 & 10 & 212 & 0,88 & $-0,13$ & & 2,27 & 0,44 & 0,03 \\
\hline Sintomáticos & 6 & 67 & 10 & 212 & 1,90 & 0,64 & & 3,57 & 1,46 & 1,47 \\
\hline Mega & 4 & 27 & 10 & 212 & 3,14 & 1,14 & & 2,56 & 3,32 & 3,67 \\
\hline ICC & 1 & 29 & 10 & 212 & 0,73 & $-0,31$ & & 0,88 & 0,08 & 0,08 \\
\hline Morte súbita & 1 & 11 & 10 & 212 & 1,9 & 0,64 & & 0,83 & 0,33 & 0,29 \\
\hline
\end{tabular}

Tabela 5 - Distribuição das freqüências dos grupos sangüineos no grupo controle, nos chagásicos assintomáticos e sintomáticos.

\begin{tabular}{|c|c|c|c|c|c|c|}
\hline & & $O$ & $A$ & $\boldsymbol{B}$ & $A B$ & TOTAL \\
\hline & Controle & $116(52,25 \%)$ & $78(35,14 \%)$ & $18(8,11 \%)$ & $10(4,50 \%)$ & 222 \\
\hline $\mathrm{C}$ & Assintomático & $40(53,33 \%)$ & $24(32,0 \%)$ & $8(10,67 \%)$ & $3(4,0 \%)$ & 75 \\
\hline A & Sintomático & $26(35,62 \%) *$ & $30(41,09 \%)$ & $11(15,07 \%)$ & $6(8,22 \%)$ & 73 \\
\hline Á & Morte súbita & $5(41,67 \%)$ & $3(25 \%)$ & $3(25 \%)$ & $1(8,33 \%)$ & 12 \\
\hline 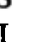 & Mega & $10(32,26 \%)^{*}$ & $13(41,94 \%)$ & $4(12,9 \%)$ & $4(12,9 \%)$ & 31 \\
\hline C & ICC & $11(36,67 \%)$ & $14(46,67 \%)$ & $4(13,33 \%)$ & $1(3,33 \%)$ & 30 \\
\hline S & Total & $66(44,59 \%)$ & $54(36,49 \%)$ & $19(12,84 \%)$ & $9(6,08 \%)$ & 148 \\
\hline
\end{tabular}

- Diferença significativa em relação ao controle $(P<0,05)$. 


\section{DISCUSSÃo}

No presente trabalho fez-se estudo estatístico comparando-se a população de chagásicos como um todo e dividida em assintomáticos, sendo estes subdivididos de acordo com os achados anatômicos e o tipo de morte em três grupos: chagásicos falecidos em ICC, chagásicos com megaesôfago e/ou megacólon e chagásicos falecidos subitamente sem ICC e sem "megas". Como material para determinação do grupo sanguineo utilizou-se simultaneamente $o$ líquido pericárdico (classificação reversa) e sangue, ambos colhidos à necrópsia. Ao utilizar apenas os casos em que os dois materiais forneceram resultados idênticos, acreditamos ter afastado as principais causas de falseamento da tipagem sanguínea. A análise estatistica foi realizada calculando-se $\mathrm{X}^{2}$ a partir de tabelas de contingência e segundo Woolf ${ }^{13}$, sendo os resultados semelhantes, observando-se apenas pequenas variações. Entretanto, para se chegar à fórmula do $\mathrm{X}^{2}$ de Woolf $\left(\mathrm{X}^{2}=\mathrm{WY}^{2}\right)$ temos de calcular " $\mathrm{x}$ ", um índice que nos dá idéia bastante precisa do quanto as duas populações comparadas diferem com relação a um fenômeno. De fato, sendo $h$ a ocorrência de um fenômeno e $\mathrm{k}$ sua ausência na população teste; $\mathrm{He} \mathrm{K}$ a ocorrência ou não do mesmo fenômeno na população controle, e sendo $\mathrm{x}=\mathrm{hK}$, o seu valor 1 indica que as

populações são idênticas quanto ao fenômeno analisado, e valores acima ou abaixo da unidade indicam que $o$ fenômeno é mais ou menos freqüente na população teste que na controle. Através deste estudo verificamos que a população de chagásicos crônicos como um todo, quando comparada com os controles não chagásicos, tem menor freqüência de indivíduos do grupo $O$ $(x=0,69)$ e maior frequêencia de pessoas dos grupos $B$ $(x=1,69)$ e $\mathrm{AB}(\mathrm{x}=1,37)$, sem que no entanto estas diferenças sejam significativas $(P>0,05)$. Entretanto, quando separamos os chagásicos assintomáticos dos sintomáticos, verificamos que nestes a freqüência do grupo $\mathrm{O}$ é significativamente menor que nos controles $(x=0,51 ; P<0,05)$. Esta menor freqüência de grupo O nos chagásicos sintomáticos é "compensada" por uma ocorrência maior dos grupos $\mathrm{B}(\mathrm{x}=2,01)$ e $\mathrm{AB}$ $(x=1,90)$, em relação aos controles. Entretanto, estas diferenças não são estatisticamente significativas. Quando analisamos o grupo de chagásicos sintomáticos com visceromegalias, verificamos também uma ocorrència significativamente menor de grupo $\mathrm{O}$ $(x=0,44 ; P<0,05)$, que se acompanha de maior freqüência dos grupos $\mathrm{B}(\mathrm{x}=1,68)$ e $\mathrm{AB}(\mathrm{x}=3,14)$ em relação aos controìes, diferenças estas não significativas $(P>0,05)$. $O$ grupo de chagásicos falecidos subitamente é pequeno, o que dificulta sua análise; mesmo assim pode-se dizer que o grupo $B$ é significativamente mais freqüente nesta população que nos controles quando se calcula o $\mathrm{X}^{2}$ por tabela de contingência, não o sendo pelo método de Woolf. Já os chagásicos com ICC têm uma menor freqüência de $O$ $(x=0,53)$ em relação ao controle, sem que no entanto seja significativa $(P>0,05)$. Estes resultados mostram que, embora os individuos necropsiados do grupo sanguíneo $O$ não sejam protegidos contra a infecção pelo $T$. cruzi, parecem relativamente protegidos quanto a sua ação patogênica, particularmente no que se refere à formação de "megas". Esta relativa resistência dos individuos do grupo O é também observada em relação a outras doenças infecciosas como giardíase ${ }^{4}$, lepra ${ }^{12}$, esquistossomose ${ }^{3} 7$ e sifilis ${ }^{11}$. Embora as conclusões baseadas em estudos estatísticos semelhantes aos citados sejam criticáveis, podem, no entender de Barbosa e cols² ${ }^{2}$ estar ligadas mais a diferenças caracterizadas por interferências raciais na suscetibilidade a determinadas doenças. Entretanto, sendo a insuficiência cardíaca mais freqüente na população de chagásicos de cor preta que na de chagásicos bran$\cos ^{8}$ era de se esperar uma maior prevalência de grupo B entre os chagásicos com ICC, o que não se verificou. Em contraposição, são os chagásicos com "megas" que têm uma freqüência significativamente menor de grupo $\mathrm{O}$ e um aumento não significativo dos grupos $\mathrm{B}$ e $\mathrm{AB}$. Como os "megas" não são mais freqüentes nos chagásicos negros ${ }^{8}$ - que é justamente a parcela da popuiação que tem, em relação aos brancos, maior prevalência de indivíduos do grupo sangüineo $\mathrm{B}^{5}$-, é pouco provável que as diferenças por nós observadas se relacionem com o fator raça. Um dos fatores admitidos como responsáveis pela influência dos grupos sanguíneos no aparecimento e evolução das doenças infecciosas é a antigenicidade cruzada entre o agente e o sistema $\mathrm{ABO}^{9}$. No caso do T. cruzi, a antigenicidade cruzada tem sido observada com alguns tecidos de mamíferos, não sendo improvável que algumas de suas cepas tenham antigenos semelhantes ao do sistema ABO. Tripanosomas portadores de antígenos semelhantes a aglutinógenos $\mathrm{A}$ ou $\mathrm{B}$ poderiam ter mais dificuldades de sobrevivência nos hospedeiros do grupo $\mathrm{O}$ que nos outros, por causa da presença de anticorpos anti-A e anti-B. Os portadores de visceromegalias apresentam maior frequência de grupos B e AB que, embora não significativa estatisticamente, estaria de acordo com a hipótese da existência de $T$. cruzi portador de antígenos semelhantes ao aglutinógeno B. Por outro lado, outros fenômenos, diversos da antigenicidade cruzada mas que influem na relação hospedeiro-parasita, poderiam acompanhar, com freqüências diversas, um ou outro grupo sanguíneo e, desta forma, tornar os portadores de determinado grupo do sistema $\mathrm{ABO}$ mais ou menos sensiveis a determinados agentes patógenos.

\section{$S U M M A R Y$}

ABO blood group distribution was studied in 222 controls and in 148 symptomatic and symptomless 
Teixeira VPA, Martins E, Almeida HO, Soares S, Souza HM, Morais CA. Sistema ABO eformas anatomoclínicas da doença de Chagas crônica. Revista da Sociedade Brasileira de Medicina Tropical 20: 163-168, Jul-Set, 1987.

patients with chronic Chagas' disease with different anatomo-clinics forms (congestive heart failure, visceromegalias and sudden death). The $X^{2}$ was estimated from contingency table and by Woolfs method. The $X^{2}$ obtained by these two methods showed that the group O frequency was significantly less in the symptomatic chagasic group as a whole and in those with "megas" when compared with the controls. B blood group was observed to be more frequent in chagasics with sudden death but the $X^{2}$ was only significant when calculated for contingency tables. The apparent relative protection confered by the $O$ blood group to chagasics with respect to its evolution to symptomatic forms of the disease, could be explained by cross antigenicity among $\mathrm{T}$. cruzi populations and $A B O$ blood group or by other factors influencing or being influenced by the blood group distribution.

Key words: Chagas' disease. Blood group. ABO blood group.

\section{REFERÊNCIAS BIBLIOGRÁFICAS}

1. Aird I, Bentall HH, Mehigan JA, Roberts JAF. The blood groups in relation to peptic ulceration and carcinoma of colon, rectum, breast and bronchus. British Medical Journal 2:315-321, 1954.

2. Barbosa CAA. Associação entre grupo sangüíneo A e esquistossomose mansônica. Revista de Saúde Pública de São Paulo 16:346-348, 1982.

3. Camus D, Bina JC, Carlier Y, Santoro F, Figueiredo JFM, Prata A, Capron A. Grupos sangüineos ABO e formas clínicas da esquistossomose mansônica. Revista do Instituto de Medicina Tropical de São Paulo 19:7779, 1977.

4. Escobar AS, Febrillet SC, Falcon JC, Borbolla E, Carbajal MG, Pomar F. Grupos sangüineos en la giardiasis. Revista Cubana de Medicina Tropical 36:88-93, 1984.

5. Henry JB. Diagnósticos clínicos em conduta terapêutica por exames laboratoriais. 16a edição, Editora Manole Ltda, São Paulo, 1983.

6. Krieger H, Barbosa CAA. Smallpox and the ABO system association: A critical review. Revista Brasileira de Biologia 39:195-199, 1979.

7. Lima Pereira FE, Bortolini ER, Carneiro JLA, Silva CRM, Neves RC. ABO blood groups and hepatosplenic form of schistosomiasis mansoni (Symmer's fibrosis) Transactions of the Royal Society Tropical Medicine and Hygiene 73:283-286, 1979.

8. Miziara LJ, Almeida HO, Chapadeiro E, Yamamoto I. Aspectos raciais dos "megas" e da cardiopatia na doença de Chagas crônica. Revista da Sociedade Brasileira de Medicina Tropical 14:1-5, 1981.

9. Oliver-González J, González LM. Release of the $\mathrm{A}_{2}$ isoagglutinogen - like substance of infectious organisms into human blood serum. Journal of Infectious Disease 85:66-71, 1949.

10. Vogel F. ABO blood groups and disease. The American Journal of Human Genetics 22:464-475, 1970.

11. Vogel F, Krüger J, Song YK, Flatz G. ABO blood groups, leprosy and serum proteins. Humangenetic 7:149-162, 1969.

12. Wiener AS: Blood groups and disease. The American Journal of Human Genetics 22: 476-483, 1970.

13. Woolf $B$. An estimating the relations between blood group and disease. Annals of Human Genetics 19:251-253, 1955. 